\title{
APLICABILIDADE DO CONCEITO TRÍPLICE BARREIRA EM MANCAIS DE TRANSPORTADORES DE CORREIA*
}

\author{
Danilo Amorin da Silva' \\ Marcelo Gonçalves. Pereira² \\ Edmilson Ricardo de Oliveira Santos ${ }^{3}$
}

\section{Resumo}

A contaminação por partículas sólidas em mancais de rolamentos de transportadores de correia é um dos principais problemas na indústria, por exemplo: mineração e preparação de madeira na produção de celulose. Este artigo mostra a Análise de Falha de Causa Raiz (RCFA) de rolamentos autocompensadores de rolos que apresentaram falha neste tipo de equipamento. Desta forma, foram identificados a presença de contaminante no interior dos mesmos, além de característica de elevação de temperatura das superfícies e graxa carbonizada. A aplicação do conceito de tríplice barreira acompanhada pela técnica análise de vibração foi essencial para o sucesso deste estudo. A montagem do conjunto contém rolamento vedado BS2-2218-2CSK/VT143, caixa de mancal SNL 518-615 e por último, preenchimento do espaço vazio interno da caixa com $90 \%$ de graxa. O resultado foi mensurado com aumento significativo do tempo entre falhas, partindo de aproximadamente 10 meses para 30 meses após a aplicação da proposta de solução.

Palavras-chave: Rolamento vedado; Tríplice barreira; Correia transportadora; Análise de vibração.

\section{APPLICABILITY OF THE TRIPLE BARRIER CONCEPT IN BELT CONVEYORS HOUSING}

\section{Abstract}

Solid particle contamination of housing on belt conveyors is one of the main problems in the industry, naming few of them: mining and cellulose - wood preparation area. This article shows the Root Cause Failure Analysis (RCFA) of selfaligning roller bearings that failed in this type of equipment. It was identified contamination inside of the bearings, as well as a characteristic of surface temperature rise and carbonized grease. The application of triple barrier concept monitored by Vibration Analysis Technique was essential for the success this study. The mounting contains the sealed bearing BS2-2218-2CSK/VT143 and housing SNL 518-615 with the housing's internal empty space filled with $90 \%$ of grease. The result was measured with a significant increase of the mean time between failures, from 10 months to 30 months after the improvement.

Keywords: Sealed Bearing; Triple barrier; Belt Conveyor; Vibration analysis.

Engenheiro Industrial Mecânico, Engenheiro de Aplicação, SKF do Brasil LTDA, SP, Brasil. Engenheiro Industrial Mecânico, Engenheiro de Aplicação, SKF do Brasil LTDA, SP, Brasil.

Engenheiro Mecânico, Engenheiro de Simulação Numérica, SKF do Brasil LTDA, SP, Brasil. 


\section{INTRODUÇÃO}

O ambiente industrial tem introduzido tecnologia e novos conceitos para uma apropriada gestão de ativos, com aumento da confiabilidade e disponibilidade de equipamentos no processo produtivo. O objetivo deste trabalho é mostrar a aplicação RCFA em rolamentos, e a aplicabilidade do conceito de tríplice barreira como melhoria em mancais, que sofrem falhas prematuras devido a contaminação de agentes sólidos. Por último, o acompanhamento da condição pela técnica preditiva Análise de Vibração.

A Figura 1 mostra rolo de transportador de correia sob condições de contaminação de particulado sólido do ambiente produtivo. Neste equipamento são montados os mancais de rolamentos autocompensadores de rolos.



Figura 1. Mancais de Transportador de Correia.

\section{MÉTODOS}

\subsection{Dados do Equipamento}

$\mathrm{Na}$ Tabela 1 estão descritos os dados do equipamento original, no qual realizou-se a avaliação do conceito de tríplice barreira.

Tabela 1. Dados da aplicação

\begin{tabular}{|c|c|}
\hline $\begin{array}{r}\text { Acionamento da Correia - } \begin{array}{r}\text { Motor } \\
\text { Potência } \\
\text { Rotacão }\end{array}\end{array}$ & $\begin{array}{l}55 \mathrm{KW}(75 \mathrm{CV}) \\
1775 \mathrm{RPM}\end{array}$ \\
\hline $\begin{array}{r}\text { Mancal Original } \\
\text { Modelo }\end{array}$ & $\begin{array}{l}\text { FCM } \\
\text { SBMA } 18\end{array}$ \\
\hline Rolamento Original & $22218 \mathrm{EK}$ \\
\hline $\begin{array}{l}\text { Lubrificação } \\
\text { Tipo } \\
\text { Especificação } \\
\text { Intervalo relubrificação } \\
\text { Quantidade }\end{array}$ & $\begin{array}{l}\text { Graxa } \\
\text { Mobilith XHP } 222 \\
30 \text { dias } \\
40 \text { gramas }\end{array}$ \\
\hline $\begin{array}{l}\text { Dados Operacionais } \\
\text { Temperatura Operacional } \\
\text { Rotação do rolo do transportador }\end{array}$ & $\begin{array}{l}50^{\circ} \mathrm{C} \text { (Condições normais) } \\
75 \text { RPM }\end{array}$ \\
\hline
\end{tabular}




\subsection{Análise de Falha de Causa Raiz (RCFA)}

A RCFA que é um processo simples e estruturado, é utilizado para investigar, corrigir e eliminar a falha do equipamento, sendo mais eficaz quando dirigido a colapsos crônicos, segundo Lepree [1].

Os rolamentos estão entre os componentes mais importantes na grande maioria de máquinas [2]. Estes componentes são mecanismos de alta tecnologia e quando ocorre falha podem ocasionar paradas não programadas em máquinas, gerando alto custo de manutenção.

Neste trabalho realizou-se a RCFA nos rolamentos de transportador de correia, classificando os modos de falha conforme a norma ISO 15243:2004 [3]. Na análise visual dos componentes, verificou-se grande quantidade de agentes contaminantes no interior dos mancais e nas partes dos rolamentos. Também observou-se a carbonização da graxa devido aquecimento excessivo após ocorrência de deficiência de lubrificação em 2013.

A Figura 2 mostra o mancal contaminado e a Figura 3 as principais características observadas na gaiola, elementos rolantes, anel interno e externo.



Figura 2. Mancal com contaminação. 

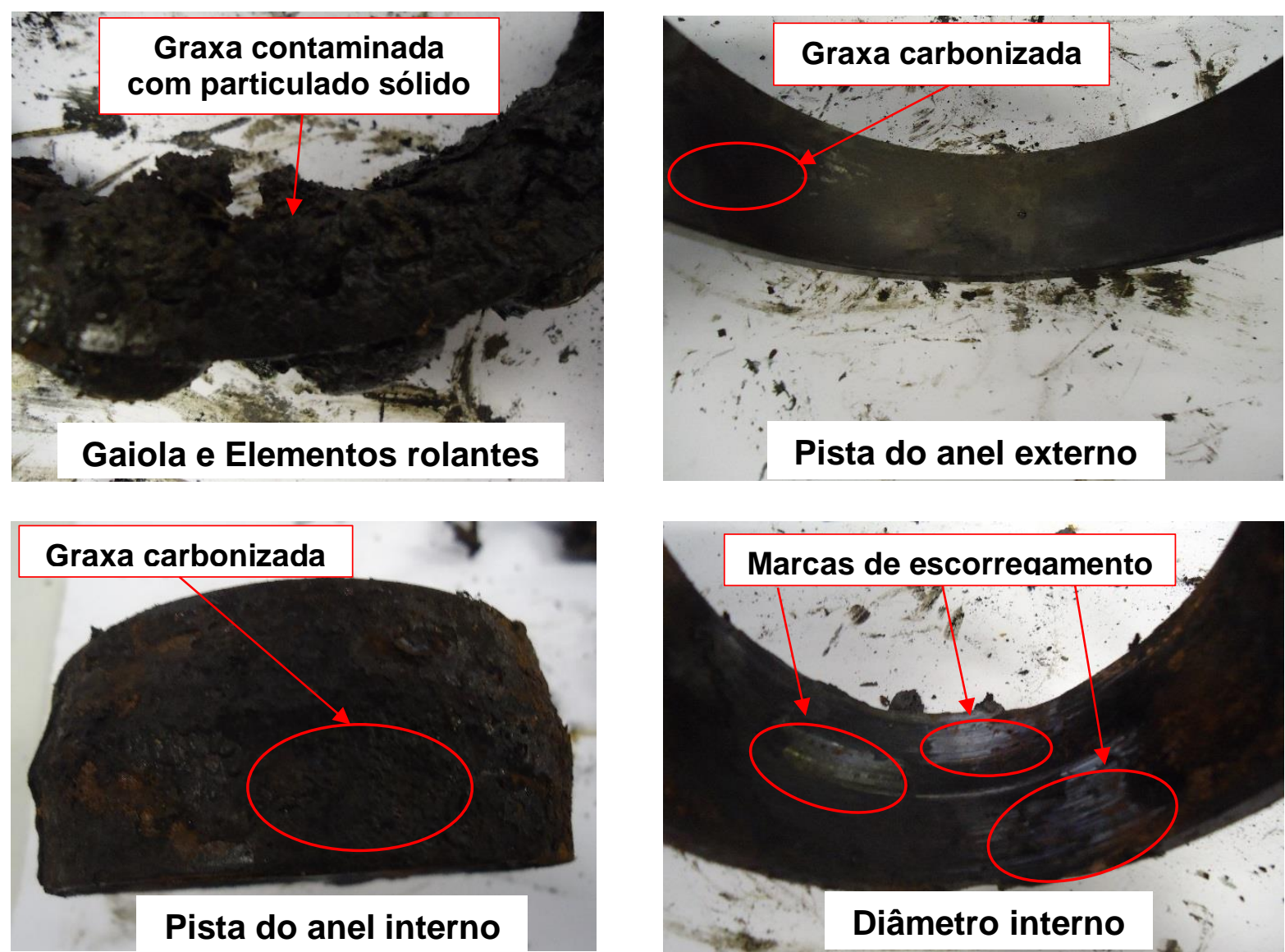

Figura 3. Partes do rolamento com contaminação e graxa carbonizada.

A trajetória de falha mais provável segundo as características observadas no rolamento analisado está apresentada na Figura 4. 


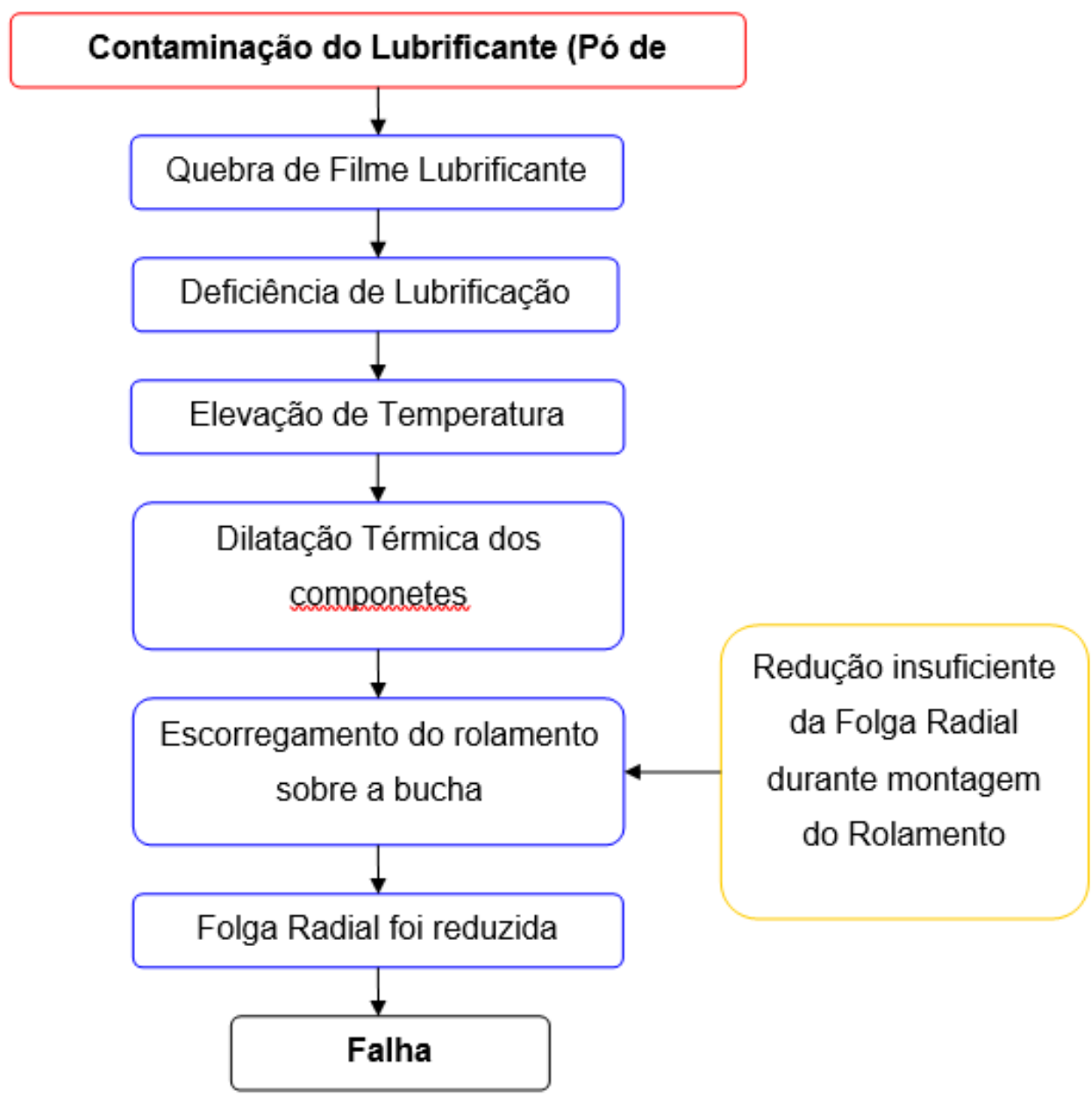

Figura 4. Trajetória de falha do rolamento analisado.

\subsection{Conceito de Tríplice Barreira}

O conceito tríplice barreira consiste em três componentes contra a entrada de contaminante [4]. O primeiro é o rolamento vedado (1), em seguida a graxa no interior do mancal (2), que deve ser preenchido em torno de $90 \%$ do espaço vazio e por último a vedação externa (3). A ilustração da Figura 5 mostra os principais componentes das barreiras, com as respectivas numerações.



Figura 5. Conceito Tríplice Barreira. (1) rolamento vedado; (2) 90\% do espaço preenchido com graxa; (3) vedação externa. 
A avaliação das condições operacionais do equipamento e a velocidade limite do rolamento vedado são indispensáveis para verificação da necessidade de relubrificação. Assim correlacionando estes parâmetros, é possível identificar as regiões 1 e 2 do diagrama da Figura 6 . A região 1 é isenta de relubrificação, já a região 2 é necessário realizar a mesma.

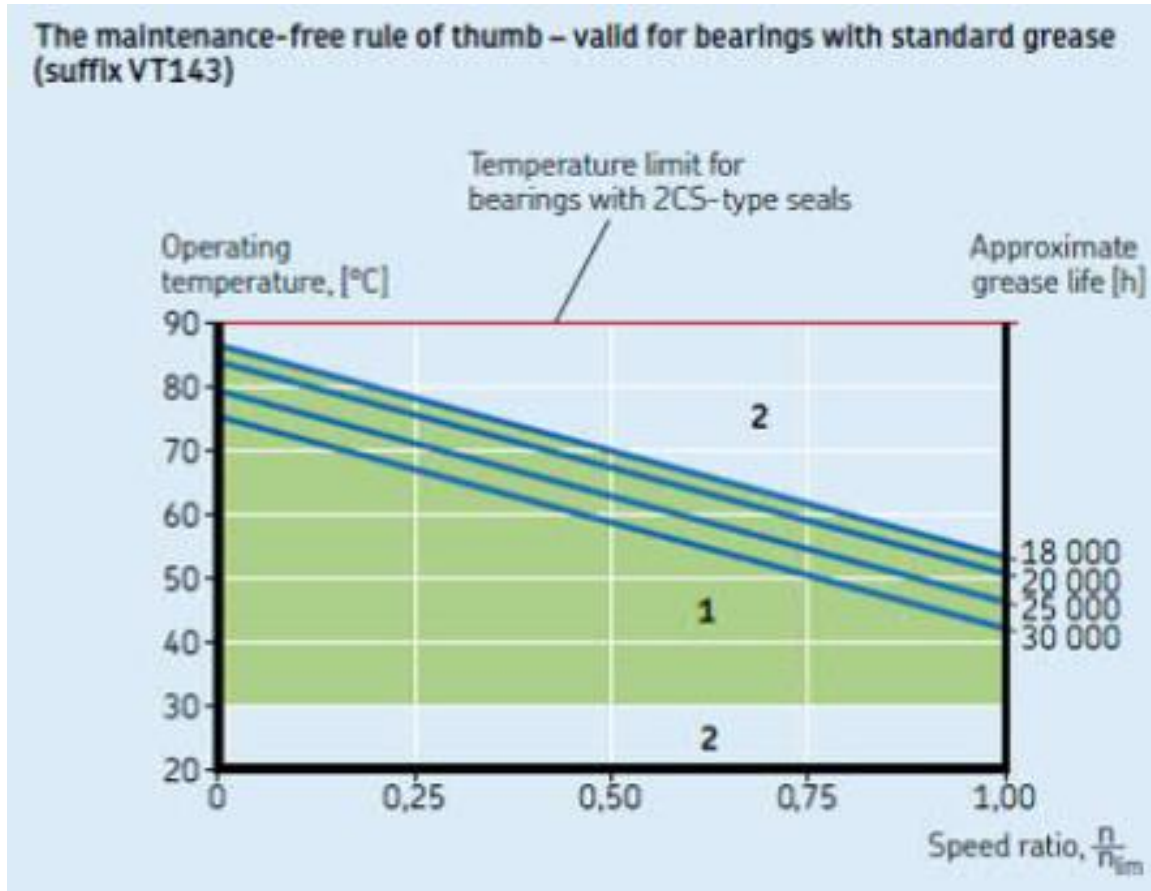

Figura 6. Diagrama análise das condições operacionais [4].

\subsection{Montagem}

A utilização de ferramentas e métodos adequados para a montagem são fatores indispensáveis para o bom funcionamento dos rolamentos. Deve-se incluir também a verificação de tolerâncias dimensionais, tais como eixo e caixa, além de verificação da planicidade da base de apoio da caixa de mancal. Neste contexto, foi indicado o método de deslocamento axial SKF Drive-up, utilizando ferramentas hidráulicas para uma montagem mais precisa dos rolamentos vedados. O método consiste na redução da folga interna radial através da aplicação de uma pressão pela bomba hidráulica e deslocamento axial pré-estabelecidos [5], conforme ilustrado na Figura 7. 


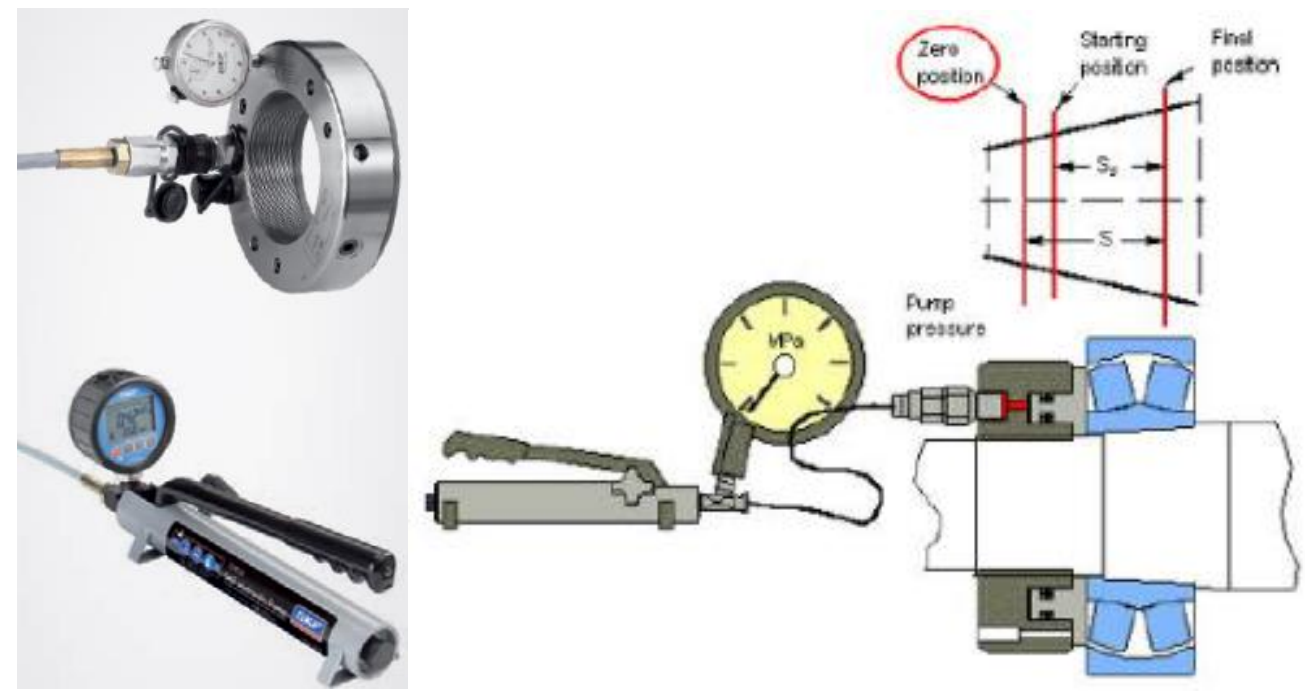

Figura 7. Método de montagem SKF Drive-up.

\subsection{Análise de Vibração}

A análise de vibração como técnica preditiva vem sendo amplamente utilizada no meio industrial como forma de gerenciar a condição de ativos rotativos. Neste contexto, foi essencial para o acompanhamento da aplicação do conceito de tríplice barreira e permitir a tomada de decisão para manutenção planejada.

No acompanhamento da condição são monitorados sinais de vibração com frequência de 30 dias em pontos de medição pré-estabelecidos, além da realização de análise dos espectros de vibração de cada um dos pontos com auxílio de um programa computacional de análise de dados.

$\mathrm{Na}$ Tabela 2 estão descritos as classificações das zonas de intensidade de vibração para equipamentos de potência acima de $15 \mathrm{~kW}$ até $300 \mathrm{~kW}$ segundo a norma ISO 10816-3 [6].

Tabela 2. Níveis de vibração para as partes rotativas

\begin{tabular}{c|c|c|c}
\hline Suporte & Zona & Deslocamento $\boldsymbol{\mu m}$ RMS & $\begin{array}{c}\text { Velocidade } \mathbf{~ m m} / \mathbf{s} \\
\text { RMS }\end{array}$ \\
\hline \multirow{3}{*}{ Rígido } & A/B & 22 & 1,4 \\
& B/C & 45 & 2,8 \\
& C/D & 71 & 4,5 \\
\hline \multirow{3}{*}{ Flexível } & A/B & 37 & 2,3 \\
& B/C & 71 & 4,5 \\
& C/D & 113 & 7,0 \\
\hline
\end{tabular}

- Zona A: As vibrações de uma nova máquina comissionada deveria normalmente cair dentro dessa zona.

- Zona B: Máquinas com vibrações dentro dessa zona são normalmente consideradas aceitáveis para um longo tempo de operação aceitável.

- Zona C: Máquinas com vibrações dentro dessa zona são normalmente consideradas não satisfatórias para operações contínuas de longo tempo. Geralmente, a máquina pode ser operada por um tempo limitado nessa condição até que apareça uma oportunidade adequada para uma ação remediada.

- Zona D: Os valores da vibração nessa zona são normalmente considerados suficientemente severos para se causar um dano em uma máquina. 
A instrumentação e programas utilizados nas medições de análise de vibração estão mostradas a seguir:

- Coletor/Analisador de dados - SKF MICROLOG GX 70.

- Sensores - Acelerômetros SKF CMSS2200.

- Programa de análise de dados e gerenciamento - SKF @ptitude Analyst 9.0.1033.1.

$\mathrm{Na}$ Figura 8 é mostrado a tendência dos níveis de vibração no parâmetro aceleração, com destaque para a medição em 25/09/2016, na qual identifica deficiência de lubrificação. O primeiro teste piloto com o rolamento vedado foi implantado em 2014, e a partir desta data acompanhou-se os dados de vibração até a identificação de deficiência de lubrificação. Em seguida foi programado e substituído por novo rolamento.

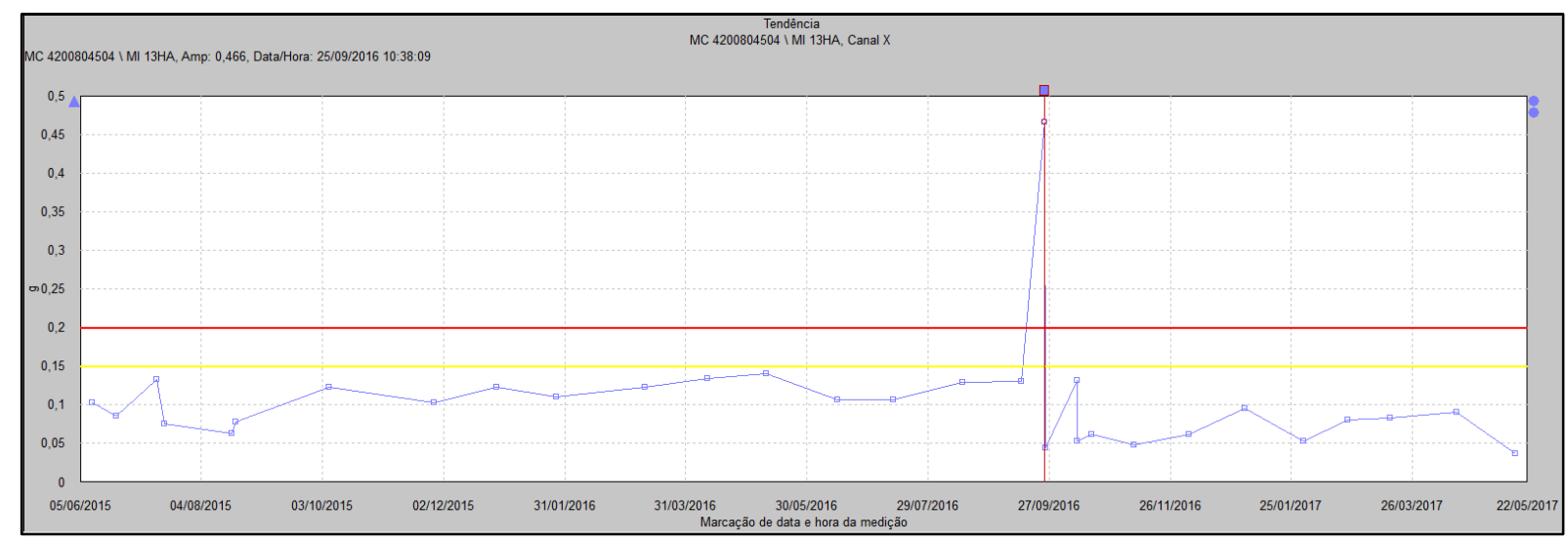

Figura 8. Tendência de vibração parâmetro aceleração.

A Figura 9 apresenta a comparação das medições realizadas antes e após a troca do rolamento. O espectro em azul caracteriza deficiência de lubrificação no rolamento e em vermelho a condição normal após a troca do mesmo. Tal comportamento anormal indica quebra de filme lubrificante, que contribui para contato metálico entre as partes internas do rolamento.

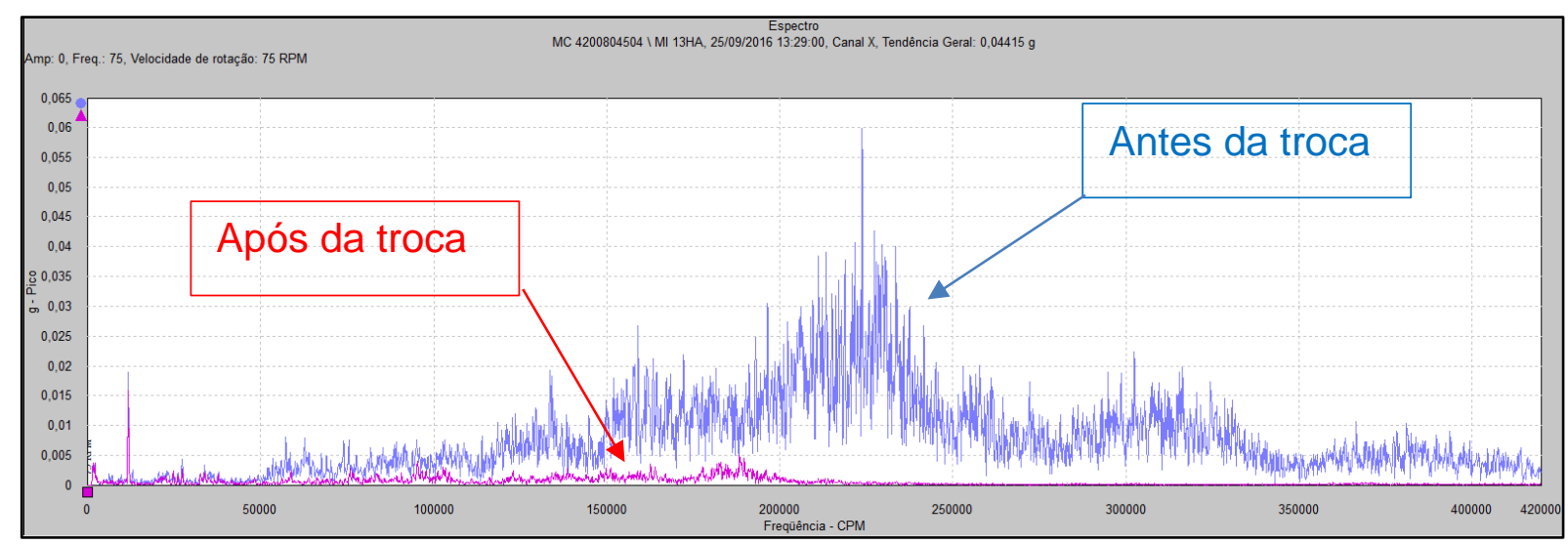

Figura 9. Espectro de vibração com comparativo de condição.

A identificação de problemas em equipamentos a partir da Análise de Vibração têm referência à Technical Associates of Charlotte [7]. 


\section{RESULTADOS E DISCUSSÃO}

A RCFA foi ferramenta fundamental para entendimento do evento de falha em mancais de rolamentos de transportadores de correias. Desta forma, aplicou-se o conceito de tríplice barreira com o objetivo de eliminar a entrada de agentes contaminantes no interior dos rolamentos. Como complemento inseriu-se uma quantidade adicional de 55 gramas de lubrificante no interior do rolamento BS2-2218-2CSK/VT143 (Figura 10), sabendo-se que originalmente ele apresenta $25 \%$ a $45 \%$ do espaço vazio interno preenchido com graxa. Outra particularidade da aplicação, é que os rolamentos operam sob condição de zona de carga na região superior do mancal, dificultando ainda mais a formação de filme lubrificante nesta região devido à gravidade. Nesta condição, com o acréscimo adicional de graxa, o espaço vazio do rolamento tornou-se de $55 \%$ a $75 \%$ preenchido, melhorando a condição de formação de filme lubrificante.

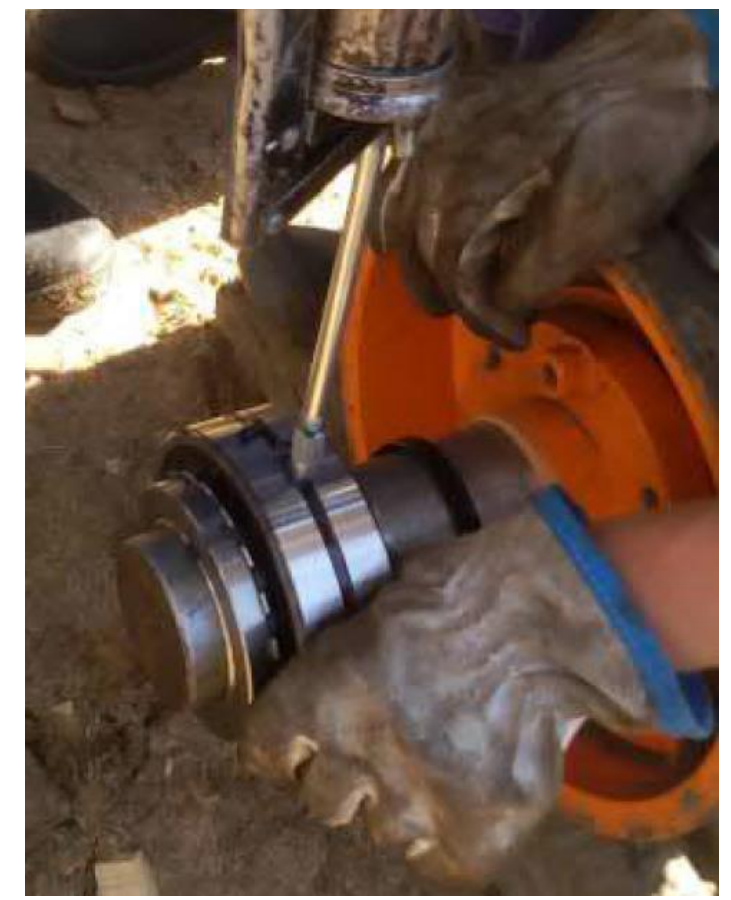

Figura 10. Acréscimo de graxa no interior do rolamento vedado.

Durante a montagem do rolamento em questão, utilizou-se o método SKF Drive-up, aplicando-se os valores da Tabela 3 que segue. Com preliminar verificação das tolerâncias dimensionais do eixo, sendo um ajuste h9 e cilindricidade IT5.

Tabela 3. Valores para aplicação do método SKF Drive-Up

\begin{tabular}{c|c|c} 
Rolamentos & $\begin{array}{c}\text { Pressão da Bomba } \\
\text { (Posição Inicial) }\end{array}$ & $\begin{array}{c}\text { Deslocamento Axial } \\
\text { (Relógio Comparador) }\end{array}$ \\
\hline SKF BS2-2218-2CSK/VT143 & $1,2 \mathrm{MPa} / 174,3 \mathrm{psi}$ & $0,538 \mathrm{~mm}$ \\
\hline
\end{tabular}

Montou-se a caixa de mancal do tipo SNL 518-615, com o preenchimento de $90 \%$ do espaço interno vazio com graxa, após o posicionamento do rolamento.

Indicou-se que o apoio da caixa de mancal deveria ter as seguintes tolerâncias:

- Planicidade: IT7

- Rugosidade da Superfície: Ra $\leq 12,5 \mu \mathrm{m}$ 
A medição dos resultados somente foi possível pelo monitoramento do equipamento através da técnica preditiva de Análise de Vibração.

A nova aplicação apresentou tempo médio entre falhas em torno de 30 meses, ao contrário da aplicação anterior que apresentava reincidências de falhas a cada 10 meses.

A programação da manutenção foi realizada de forma preventiva, já que foram identificados elevação dos níveis de vibração acima dos limites pré-estabelecidos de alerta e alarme. No entanto, em análise posterior dos rolamentos vedados, verificou-se que poderiam continuar em operação por maior período.

Os rolamentos vedados desmontados foram reutilizados em equipamentos menos críticos, retirando-se as vedações e adotando-se a relubrificação através do canal de lubrificação W33 do rolamento, conforme ilustrada na Figura 11.

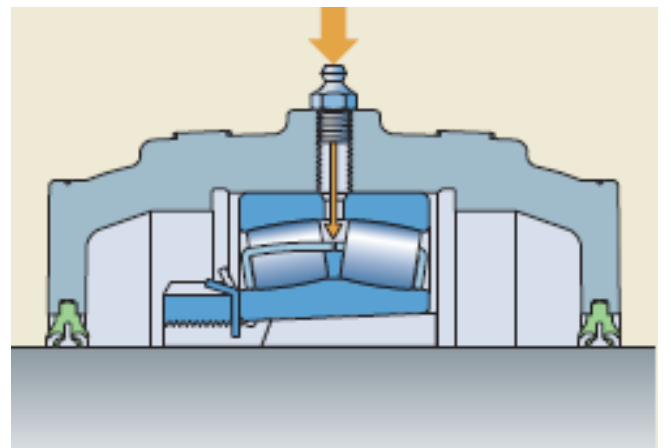

Figura 11. Canal de lubrificação W33 do rolamento.

\section{CONCLUSÃo}

No contexto de ambiente produtivo é indispensável a gestão do ativo para prévia programação de manutenção. Diante disto, neste trabalho adotou-se a RCFA como parte inicial de identificação de falha, em seguida aplicação de conceito tríplice barreira como contenção da causa raiz e por último o acompanhamento preditivo através de análise de vibração como parte integrante de controle e validação da melhoria aplicada.

Os custos de manutenção foram reduzidos significativamente, onde anteriormente havia falhas a cada 10 meses, agora atingiu a marca de 30 meses de operação. Além dos ganhos quanto ao processo produtivo pela maior disponibilidade do equipamento.

\section{REFERÊNCIAS}

1 Lepree, J. RCFA: Root Causes Failure Analysis. Disponível em: <http://www.tarrani.net/linda/RootCauseAnalysis.pdf >. Acesso em: 10 jun. 2017.

2 SKF, Bearing damage and failure analysis, $14219 \mathrm{EN}$, published 2014.

$3 \quad$ Norma ISO 15243:2004 - Rolling bearings - Damage and failures, 2004.

4 Sealed SKF Explorer Spherical Roller Bearings, Optimum protection against contaminants, downtime and high maintenance costs, 28 Pages, april 2013.

5 Silva, D. A.; Massoti, J.G.B.; Pereira, M.G. Eliminação de Falhas Prematuras de Rolamentos de Transportador Aplicando o Conceito Tríplice Barreira. Revista O Papel edição Novembro 2016, p. 83-84.

6 Norma ISO 10816-3, "Mechanical vibration - Evaluation of machine vibration by measurements on nonrotating parts", 1998. 
7 Technical Associates of Charlotte, "Use of Vibration Signature Analysis to Diagnose Machine Problems”, Chapter 6, 1997. 